\title{
Valores normativos del sistema internacional de imágenes afectivas en población mexicana: diferencias entre Estados Unidos, Colombia y México*
}

Normative Values of the International Affective Picture System in Mexicans: Differences between USA, Colombia and Mexico

\author{
TANIA RoMo-GonzÁLEZ \\ Universidad Veracruzana, México \\ ORCID: http://orcid.org/0000-0003-0097-4780 \\ Raquel González-Ochoa \\ Universidad Veracruzana, México \\ Carlos Gantiva \\ Universidad de San Buenaventura, Colombia \\ Yolanda CAMPOS-UsCANGA \\ Universidad Veracruzana, México
}

a Autor de correspondencia. Correo electrónico: tromogonzalez@uv.mx

Para citar este artículo: Romo-González, T., GonzálezOchoa, R., Gantiva, C., \& Campos-Uscanga, Y. (2018). Valores normativos del sistema internacional de imágenes afectivas en población mexicana: diferencias entre Estados Unidos, Colombia y México. Universitas Psychologica, 17(2), 1-9. https://doi.org/10.11144/Javeriana.upsy.17-2.vnsi

\section{RESUMEN}

Uno de los instrumentos con mayor evidencia empírica y versatilidad para el estudio de las emociones y la motivación es el Sistema Internacional de Imágenes Afectivas (International Affective Picture System, IAPS). Sin embargo, no ha sido probado en población mexicana. Por esta razón el presente estudio tuvo por objetivo determinar los valores normativos en valencia, arousal y dominancia para los conjuntos 13, 14, 19 y 20 del IAPS en población mexicana, e identificar las diferencias con países de América (Colombia y Estados Unidos). Participaron 408 estudiantes quienes evaluaron 238 imágenes del IAPS a través del Self-Assessment Manikin (SAM). Los resultados mostraron que la distribución de las imágenes en el espacio afectivo bidimensional (valencia-arousal) adoptan la forma esperada de boomerang, coincidiendo con lo encontrado en otras poblaciones. Sin embargo, en población mexicana es más fuerte la relación entre valencia apetitiva y arousal que entre valencia aversiva y arousal. En conclusión, los valores normativos de los conjuntos 13, 14, 19 y 20 pueden ser utilizados en población mexicana para el estudio objetivo de las emociones.

Palabras clave

sistema Internacional de Imágenes Afectivas; valencia; arousal; dominancia; emociones.

\section{ABSTRACT}

The International Affective Picture System (IAPS) is an instrument to the study of emotions and motivation with a high empirical evidence and versatility. However, it has not been tested in Mexican population, for that reason the aim of this study was to determine the normative values of valence, arousal and dominance for the IAPS sets of pictures 13, 14, 19 and 20 in Mexican population. Participants were 408 students who 
assessed 238 images of the IAPS through the SelfAssessment Manikin (SAM). The results showed that the pictures distribution on the two-dimensional affective space (valence-arousal); take a boomerang form, which is consistent with the findings in other populations. Nevertheless, in the Mexican population there was a stronger relationship between appetitive valence and arousal than the one observe in aversive valence and arousal. In conclusion the normative values in the IAPS sets of pictures 13,14, 19 and 20 can be used in the study of emotions in Mexican people.

Keywords

international Affective Picture System; valence; arousal dominance; emotions.

El estudio científico de las emociones requiere instrumentos válidos y confiables que permitan estudiar la respuesta emocional y de sus componentes de forma objetiva. Uno de los instrumentos con mayor evidencia empírica y versatilidad para el estudio de las emociones y la motivación es el Sistema Internacional de Imágenes Afectivas (International Affective Picture System, IAPS) (Lang, Bradley, \& Cuthbert, 1999; Lang, Bradley, \& Cuthbert, 2008). El IAPS está compuesto por más de 1000 imágenes a color que abarcan una gran variedad de áreas de la vida diaria de las personas, como los deportes, la familia, el sexo, los animales, el trabajo, accidentes, comida, el hogar, la violencia, entre otras. Las imágenes están distribuidas en 20 conjuntos de 60 imágenes cada uno, en promedio.

El IAPS está basado en el modelo bioinformacional de las emociones (Lang, 1995; Lang, 2010), el cual concibe a la emoción como una predisposición para la acción a partir de la activación de uno de los dos sistemas motivacionales primarios, los cuales han evolucionado para mediar los cambios ambientales que pueden ser amenazantes o que promueven la supervivencia de un individuo (Bradley, Codispoti, Cuthbert, \& Lang, 2001; Bradley \& Lang, 2000; Lang, Bradley, \& Cuthbert, 1997).

Los sistemas motivacionales primarios son el defensivo y apetitivo; el defensivo se activa ante contextos relacionados con la amenaza que generan conductas de escape, retirada y ataque, mientras que el sistema apetitivo se activa en situaciones que promueven la supervivencia (sustento, procreación y crianza), basándose en conductas de ingestión, cópula y prestación de cuidados (Bradley \& Lang, 2007). La activación de cualquiera de estos sistemas da inicio a un conjunto de respuestas atencionales y motoras, tales como el reflejo de orientación, la atención mantenida (acompañada de la bradicardia del miedo), la movilización de recursos y la preparación para la acción (aumento de la respuesta galvánica de la piel y del reflejo de sobresalto), que facilitan la selección del comportamiento adaptativo (Bradley, 2009).

El modelo bio-informacional organiza la experiencia emocional en tres niveles jerárquicos; en el primer nivel se encuentran los patrones específicos de la respuesta emocional, los cuales dependen del contexto en el que se presentan y que poseen patrones conductuales y fisiológicos concretos y delimitados. En el nivel intermedio se encuentran las emociones, tal como las conocemos, las cuales están compuestas por subrutinas de ataque, lucha, huida, aproximación, conducta sexual y búsqueda de alimento, entre otras, que originan estereotipias de respuesta ante diversas situaciones. Finalmente, en el nivel superior, se encuentran las dimensiones que son compartidas por todas las emociones: valencia, arousal y dominancia (Bradley et al., 2001; Gantiva, Guerra, \& Vila, 2011; Vila \& Fernández-Santaella, 2005; Vila et al., 2001).

La valencia es la dimensión sobre la cual se estructura la respuesta emocional, está determinada por la activación de uno de los dos sistemas motivacionales primarios: el apetitivo, el cual se manifiesta a través de conductas de aproximación, y el defensivo a través de conductas de escape y evitación (Lang, 1995). La valencia posee un sustrato neurofisiológico específico para cada sistema motivacional, por lo cual es la dimensión que mayor influencia la respuesta emocional (Lang \& Davis, 2006; LeDoux, 2000) y refleja el grado de placer o displacer experimentado por la persona ante una situación particular (Gantiva et al., 2011). 
El arousal o activación hace referencia al nivel de energía invertido en la emoción y representa la activación metabólica y neural de cualquiera de los sistemas, ya sea apetitivo o defensivo (Bradley, 2009). Finalmente, la dominancia es la dimensión encargada de explicar el grado de control percibido sobre la respuesta emocional y a su vez implica la interrupción o continuidad de la respuesta conductual (Gantiva et al., 2011; Vila et al., 2001).

El IAPS es uno de los principales instrumentos para el estudio objetivo de las dimensiones de la emoción, se ha encontrado en diferentes países y poblaciones que el uso del IAPS para la investigación científica de las emociones resulta confiable y válido. En los últimos años se ha demostrado que los estímulos que conforman el IAPS tienden a comportarse de forma similar en las diferentes poblaciones en las cuales se ha aplicado, por ejemplo en población estadounidense (Lang et al., 1999; Lang et al., 2008), española (Moltó et al., 1999; Vila et al., 2001), belga (Verschuere, Crombez, \& Koster, 2001), colombiana (Gantiva et al., 2011) y chilena (Dufey, Fernández, \& Mayol, 2011; Silva, 2011). En población mexicana se han utilizado algunas imágenes del IAPS para el estudio de respuestas emocionales, pero no se obtuvieron los valores normativos en las dimensiones de valencia, arousal y dominancia (Chayo-Dichy, Velez, Arias, Castillo-Parra, \& Ostrosky-Solis, 2003). Por lo anterior, el objetivo de la presente investigación fue establecer los valores normativos en valencia, arousal y dominancia de cuatro conjuntos del IAPS (238 imágenes) para generar emociones en población mexicana. Los resultados de esta investigación permitirán utilizar estas imágenes del IAPS para estudiar la emoción y la motivación en población mexicana.

\section{Método}

\section{Participantes}

El estudio se llevó a cabo con 408 estudiantes (188 hombres y 220 mujeres) de cuatro facultades de la Universidad Veracruzana, en México, procedentes de diversos lugares del país, con edades entre los 17 y 37 años $(M=19.81$ y $D E=2.58)$, elegidos a través de un muestreo no probabilístico. Se informó a cada uno de los participantes el objetivo de la investigación y se les entregó un formato de consentimiento informado dándoles la oportunidad de decidir si querían o no participar en el estudio.

\section{Instrumentos}

International Affective Picture System. El IAPS está conformado por más de 1000 imágenes a color, estas se encuentran agrupadas en 20 conjuntos, cada uno de ellos con un promedio de 60 imágenes que representan todas las posibles combinaciones de las dimensiones que conforman las emociones (Lang et al., 2008). Se utilizaron 238 imágenes a color pertenecientes a los conjuntos 13, 14, 19 y 20 del IAPS presentadas en diapositivas a través de video beam en espacios cerrados. Los conjuntos 13 y 14 están conformados por 60 imágenes cada uno y los conjuntos 19 y 20 por 59. Los contenidos en las fotos muestran un amplio abanico de categorías (deporte, comida, sexo, violencia, desastres naturales, animales, neutrales, entre otras).

Self-Assessment Manikin (SAM). El SAM es un instrumento de autorreporte diseñado por Bradley y Lang (1994), para evaluar las dimensiones de la emoción. Este instrumento consta de tres grupos de pictogramas con figura humanoide, uno para cada dimensión de la emoción. Cada grupo consta de 5 dibujos y cuatro espacios entre ellos, lo cual permite al sujeto moverse en un rango de 9 puntos por dimensión. Para la valencia los dibujos van desde un muñeco con una sonrisa amplia que indica agrado, hasta un muñeco con una mueca de enfado. En la dimensión de arousal el grupo de pictogramas va desde un muñeco que aparece tembloroso y con un estallido en su cuerpo, hasta el extremo donde el muñeco aparece con los ojos cerrados en un estado de calma. Por último, para la dominancia, 
va desde un muñeco muy pequeño hasta uno muy grande que representa el nivel de control.

\section{Procedimiento}

Se utilizaron instrucciones grabadas en audio para explicar el objetivo y la manera de evaluar las imágenes, así como cinco imágenes de prueba que les permitió a los participantes practicar la calificación en el SAM (Lang et al., 1999).

La presentación de las imágenes, las instrucciones y el tamaño de la muestra se realizaron siguiendo estrictamente el protocolo desarrollado por Lang et al. (2008). Para la aplicación de los conjuntos se formaron grupos de 25 personas aproximadamente, para obtener un total de 100 personas como mínimo para la baremación de cada conjunto. Se controló que la proporción de hombres y mujeres no fuera superior a 1:2 o a 2:1. La aplicación se hizo de forma grupal, presentando primero el consentimiento informado y luego las instrucciones grabadas en audio-video para evitar diferencias en la entrega de la información (Gantiva et al., 2011).

La secuencia para la presentación de cada ensayo de imágenes inició con una diapositiva de preparación durante 5 segundos que decía "Evalúe la siguiente diapositiva en la fila X", inmediatamente después se presentó durante 6 segundos la imagen que debe ser evaluada. Finalmente, se proyectó una diapositiva que indicaba la evaluación durante 15 segundos: "Por favor, evalúe la diapositiva en las tres dimensiones", tiempo en el cual cada persona lo hace en el SAM.

\section{Análisis estadístico}

Una vez tabuladas las valoraciones de las dimensiones de la emoción por cada imagen o conjunto de imágenes, se hizo el análisis de datos utilizando el programa estadístico SPSS versión 20.0 para Windows. Inicialmente se presentan las medias y desviaciones estándar para el total de las imágenes y las correlaciones de Pearson entre las dimensiones de la emoción; posteriormente se presenta la distribución de las imágenes en el espacio afectivo bidimensional (valencia/arousal) a través de diagramas de puntos y las correlaciones lineales y cuadráticas entre valencia total y valencia positiva $y$ aversiva con respecto al arousal. Finalmente, para evaluar el efecto del sexo y la cultura sobre las tres dimensiones de la emoción, se realizaron tres ANOVAs $3 \times 2$ mixtas para medias repetidas, como variable intrasujeto, "País" (México, EE.UU. y Colombia); como variable intersujeto, "Sexo"; y como variables dependientes, valencia, arousal y dominancia. La corrección Greenhouse-Geisser se utilizó para corregir cualquier violación de la esfericidad en el factor de medidas repetidas. Los análisis post-hoc se realizaron con la corrección Bonferroni para comparación de pares, el tamaño del efecto $\left(\eta^{2} \mathrm{p}\right)$ también se presenta. La significancia para todos los análisis se estableció en 0.05 .

\section{Resultados}

De acuerdo con su valoración en las tres dimensiones, no se observaron diferencias estadísticamente significativas entre hombres y mujeres en las dimensiones de valencia y arousal, pero sí en dominancia, en donde los hombres tuvieron mayor puntaje que las mujeres ( $t$ (474) $\left.=3.37, p=0.001, \eta^{2} \mathrm{p}=0.023\right)($ Tabla 1$)$.

\section{Tabla 1}

Medias y desviaciones estándar de los conjuntos 13, 14, 19 y 20 del IAPS por dimensiones en población mexicana

\begin{tabular}{lcccccc}
\hline & \multicolumn{2}{c}{ Total } & \multicolumn{2}{c}{ Hombres } & \multicolumn{2}{c}{ Mujeres } \\
\cline { 2 - 7 } & $M$ & $D E$ & $M$ & $D E$ & $M$ & $D E$ \\
\hline Valencia & 5.31 & 1.62 & 5.42 & 1.45 & 5.21 & 1.88 \\
Arousal & 4.69 & 0.89 & 4.66 & 1.02 & 4.73 & 0.93 \\
Dominancia & 5.52 & 0.85 & $5.68 *$ & 0.79 & $5.4 *$ & 0.99 \\
\hline & \multicolumn{3}{c}{${ }^{*} p=0.001$} & & &
\end{tabular}

Todas las dimensiones mostraron correlaciones positivas estadísticamente significativas entre ellas para la población total y para hombres y mujeres, a excepción de la relación entre dominancia y arousal en las mujeres (Tabla 2). 
Tabla 2

Correlación lineal de Pearson para las tres dimensiones de la emoción en la muestra total y por sexo

\begin{tabular}{clcccccc}
\hline & & \multicolumn{3}{c}{ Arousal } & \multicolumn{3}{c}{ Dominancia } \\
\cline { 3 - 7 } & & MT & M & H & MT & M & H \\
\hline \multirow{2}{*}{ Valenciay } & MT & $0.47^{* *}$ & & & $0.86^{* *}$ & & \\
& H & & $0.41^{* *}$ & & & $0.85^{* *}$ & \\
& H & & $0.58^{* *}$ & & & $0.8^{* *}$ \\
\hline \multirow{2}{*}{ Arousal } & MT & & & & $0.19^{*}$ & & \\
& H & & & & & 0.07 & \\
& & & & & & $0.36^{* *}$ \\
\hline
\end{tabular}

Nota. MT: Muestra Total. M: Mujeres.

$\mathrm{H}:$ Hombres $* p<0.05, * * p<0.001$

Distribución de las imágenes en el espacio afectivo bidimensional en la población mexicana

En el espacio afectivo bidimensional conformado por valencia (eje vertical) y arousal (eje horizontal) se observa que las imágenes adoptan la forma tradicional de boomerang en donde el origen se encuentra en el punto neutro de valencia y de baja activación, y los dos extremos se extienden cada uno hacia el polo positivo (valencia apetitiva y alto arousal) y el polo negativo (valencia aversiva y alto arousal) (Figura $1)$.

\section{Figura 1}

Distribución de las 238 imágenes del IAPS en las dimensiones de valencia y arousal en población mexicana

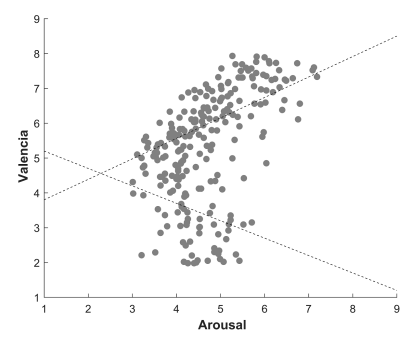

También se observa que el brazo que se extiende hacia el polo positivo tiene mayor inclinación y menor dispersión en comparación con el brazo que se extiende hacia el polo negativo, y que existen lugares en el espacio afectivo bidimensional en donde no se ubicó ninguna imagen, esto concuerda con la correlación cuadrática entre los valores de valencia y arousal, la cual es positiva y significativa $(R=0.47, p<0.0001)$.
Semejanzas y diferencias entre hombres y mujeres en la valoración de las imágenes

En el caso de los hombres, el brazo que se extiende hacia el polo positivo es el que presenta mayor inclinación y menor dispersión $(r=0.68$, $p<0.0001$ ), en comparación con el brazo que se extiende hacia el polo negativo $(r=-0.22, p=$ 0.03) (Figura 2).

\section{Figura 2}

Distribución de las 238 imágenes del IAPS en las dimensiones de valencia y arousal en hombres mexicanos

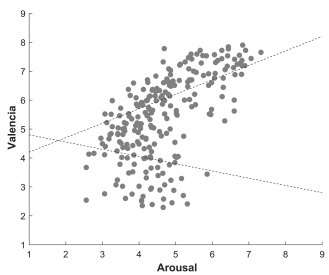

En las mujeres también hubo mayor inclinación y menor dispersión en el polo positivo $(r=0.77, p<0.0001)$ en comparación con el polo negativo $(r=-0.54, p<0.0001)$ (Figura $3)$. Por lo anterior, tanto para hombres como para mujeres mexicanas la relación entre valencia y arousal es más fuerte para la estimulación apetitiva.

\section{Figura 3}

Distribución de las medias de las 238 imágenes del IAPS evaluadas por mujeres mexicanas

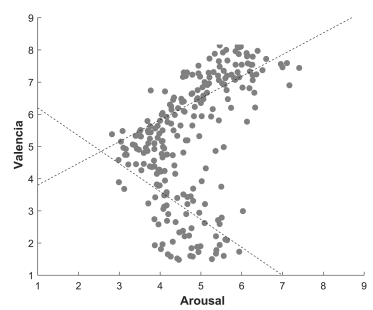

Comparación por países y sexo

Valencia: El ANOVA para valencia encontró un efecto principal significativo para "País" (F $(2,94)=53.6, p<0.0001, \eta^{2} \mathrm{p}=$ 0.1). La población mexicana tuvo valores significativamente superiores en valencia, en comparación con la población colombiana $(\mathrm{M}=$ 5.11) y con la población estadounidense $(M=5)$ 
(todas las $p<0.01$ ). No se encontró un efecto significativo para "Sexo" ni para la interacción "País" $\times$ "Sexo" (todas las $p>0.1$ ).

Arousal: El ANOVA para el arousal encontró un efecto principal significativo para "País" ( $F$ $(2,94)=33.35, p<0.0001, \eta^{2} \mathrm{p}=$ 0.06). La población mexicana tuvo valores significativamente inferiores en arousal en comparación con la población colombiana (M $=4.99)$ y con la población estadounidense $(\mathrm{M}$ $=4.83$ ) (todas las $p<0.01$ ). Se identificó un efecto principal significativo para "Sexo" (F (1, $\left.47)=4.81, p=0.02, \eta^{2} p=0.01\right)$, las mujeres tuvieron un mayor puntaje en arousal que los hombres $(M=4.94$ vs. $M=4.74 ; p=0.02)$. También se encontró un efecto de interacción significativo entre "País" $\times$ "Sexo" $(F(2,94)$ $\left.=6.46, p=0.003, \eta^{2} \mathrm{p}=0.01\right)$, las mujeres mexicanas tuvieron menores puntajes que las mujeres colombianas $(M=5.16 ; p<0.0001)$ y que las mujeres estadounidenses $(M=4.92 ; p=$ $0.01)$, y los hombres mexicanos menores puntajes que los colombianos $(M=4.83 ; p=0.001)$.

Dominancia: El ANOVA para dominancia encontró un efecto principal significativo para "País" $\left(F \quad(2,94)=80.98, p<0.0001, \eta^{2}\right.$ p $=0.14)$. La población mexicana tuvo valores significativamente superiores en dominancia en comparación con la población colombiana $(\mathrm{M}=5.46 ; p=0.02)$ y con la población estadounidense $(M=5.19 ; p<0.0001)$. Se identificó un efecto principal significativo para "Sexo" $(F(1,47)=4.82, p=0.02, \eta 2 \mathrm{p}=$ 0.01 ), los hombres tuvieron mayor puntaje en dominancia que las mujeres $(M=5.48$ vs. $M$ $=5.31 ; p=0.02$ ). También se encontró un efecto de interacción significativo entre "País" $\times$ "Sexo" $(F(2,94)=47.83, p<0$.0001, $\left.\eta^{2} \mathrm{p}=0.09\right)$, las mujeres mexicanas tuvieron menores puntajes que las colombianas $(\mathrm{M}=$ 5.54; $p=0.001)$, pero mayores puntajes que las estadounidenses $(M=5 ; p<0.0001)$, y los hombres mexicanos mayores puntajes que los colombianos $(M=5.39)$ y los estadounidenses $(M=5.38)$ (todas las $p<0.0001)$.

Las correlaciones entre población mexicana y población colombiana y estadounidense fueron positivas y significativas en valencia, arousal y dominancia para el total de la población y para hombres y mujeres por separado (Tabla 3).

\section{Tabla 3}

Correlaciones lineales para población mexicana, colombiana y estadounidense

\begin{tabular}{|c|c|c|c|c|c|c|c|}
\hline \multirow{5}{*}{$\begin{array}{l}\text { Valencia } \\
\text { México }\end{array}$} & \multirow{5}{*}{$\begin{array}{l}\text { Total } \\
\text { Hombres } \\
\text { Mujeres }\end{array}$} & \multicolumn{3}{|c|}{ Valencia Colombia } & \multicolumn{3}{|c|}{ Valencia EE.UU. } \\
\hline & & Total & Hombres & Mujeres & Total & Hombres & Mujeres \\
\hline & & $0.94 * *$ & \multirow{3}{*}{$0.88^{*}$} & & $0.93 * *$ & \multirow{3}{*}{$0.88^{*}$} & \\
\hline & & & & & & & \\
\hline & & & & $0.94 * *$ & & & $0.94 * *$ \\
\hline \multirow{7}{*}{$\begin{array}{l}\text { Arousal } \\
\text { México }\end{array}$} & & \multicolumn{3}{|c|}{ Arousal Colombia } & \multicolumn{3}{|c|}{ Arousal USA } \\
\hline & & Total & Hombres & Mujeres & Total & Hombres & Mujeres \\
\hline & & $0.78^{* *}$ & \multirow{3}{*}{$0.81^{* * *}$} & & $0.6^{* *}$ & \multirow{3}{*}{$0.66^{* * *}$} & \\
\hline & Hombres & & & & & & \\
\hline & Mujeres & & & $0.69^{* *}$ & & & $0.56^{* *}$ \\
\hline & & \multicolumn{3}{|c|}{ Dominancia Colombia } & \multicolumn{3}{|c|}{ Dominancia USA } \\
\hline & & Total & Hombres & Mujeres & Total & Hombres & Mujeres \\
\hline \multirow{3}{*}{$\begin{array}{l}\text { Dominancia } \\
\text { México }\end{array}$} & Total & $0.87 * *$ & \multirow{3}{*}{$0.69 * *$} & & $0.8^{* * *}$ & \multirow{3}{*}{$0.64 * *$} & \\
\hline & Hombres & & & & & & \\
\hline & Mujeres & & & $0.84 * *$ & & & $0.82 * *$ \\
\hline
\end{tabular}

\section{Discusión}

El objetivo de esta investigación fue el establecimiento de los valores normativos para cuatro conjuntos de imágenes del IAPS para generar emociones en población mexicana y la comparación de estos valores normativos con los obtenidos en Colombia y Estados Unidos. Los resultados indican que las imágenes de los conjuntos 13, 14, 19 y 20 del IAPS generan una respuesta en las dimensiones de la emoción similar a la encontrada originalmente por Lang et al. (1999; 2008), en donde las imágenes con valencia apetitiva generan alto arousal al igual que las aversivas; por el contrario las imágenes con valencia neutral generan bajo arousal, y adoptan la forma de boomerang, la cual ha sido reportada en los diferentes países y culturas en donde el IAPS se ha adaptado, Colombia (Gantiva et al., 2011), Estados Unidos (Bradley \& Lang, 2007), Bélgica (Verschuere et al., 2001), España (Moltó et al., 1999; Vila et al., 2001) y Chile (Dufey et al., 2011; Silva, 2011). Esto es similar a lo observado en la vida cotidiana donde lo agradable y aversivo genera una respuesta inmediata de activación, mientras que lo neutral puede resultar indiferente.

Los resultados también indican que en población mexicana es más fuerte la relación entre valencia apetitiva y arousal que entre valencia aversiva y arousal, esto se observa 
en los valores de las correlaciones y en la forma que adopta la Figura 1 en donde el polo positivo (valencia apetitiva y alto arousal) es más pronunciado y menos disperso que el polo negativo (valencia aversiva y alto arousal), estos mismos resultados se repiten cuando se divide la muestra en hombres y mujeres. Estos datos difieren de lo encontrado por Lang et al. (1999; 2008) en población general, en donde se identificó un sesgo hacia el polo negativo, mostrando una mayor relación entre las imágenes aversivas y el incremento en el arousal. No obstante, cuando los datos se dividen por sexo, los resultados en población norteamericana muestran que el sesgo hacia el polo positivo es característico en los hombres (Bradley, Codispoti, Sabatinelli, \& Lang, 2001), sin embargo, en población mexicana este sesgo también se observó en las mujeres.

Las diferencias encontradas entre países sugieren nuevamente un sesgo hacia el polo positivo en población mexicana en comparación con población colombiana y estadounidense, lo que se refleja también en los puntajes inferiores en arousal y los puntajes superiores en dominancia en población mexicana. Estas diferencias en los resultados pueden ser explicadas por el tipo y la cantidad de imágenes utilizadas en el presente estudio, pues es posible que las imágenes desagradables de los conjuntos $13,14,19$ y 20 sean menos aversivas e intensas que las de los otros conjuntos utilizados por los estudios originales de Lang et al. (1999; 2008) y Bradley et al. (2001). Sin embargo, también es posible que estas diferencias se deban a factores sociales y de habituación, pues una de la facultades de las que se tomó la muestra fue enfermería y estos estudiantes tienen contacto frecuente con imágenes de cuerpos con enfermedades y mutilación, los cuales son contenidos frecuentes de las imágenes desagradables del IAPS, (Bradley et al., 2001; Bradley et al., 2001) lo que explicaría la ausencia del sesgo negativo en las mujeres. Otra posible explicación a estos resultados en mujeres mexicanas puede ser el factor cultural, ya que de acuerdo a lo reportado por la Organización de Cooperación y Desarrollo Económicos (OCDE,
2014), México ocupa a nivel mundial los primeros lugares en cuanto a felicidad y bienestar percibido. En este reporte, el $82 \%$ de la población mexicana señala estar satisfecha con su vida, es decir manifiestan tener más experiencias positivas en un día normal (satisfacción por sus logros, sentimientos de paz, gozo, etc.) que experiencias negativas (dolor, preocupación, aburrimiento, etc.) (OCDE, 2014).

Los resultados también muestran que en población mexicana la dominancia aumenta ante imágenes con valencia apetitiva, pero disminuye ante imágenes con valencia aversiva, esto se cumple para el total de la población y para hombres y mujeres por separado. Estos mismos resultados han sido encontrados en diferentes poblaciones (Bradley et al., 2001; Gantiva et al., 2011; Lang et al., 1999; Lang et al., 2008), lo que aumenta la evidencia con relación a la primacía de la estimulación aversiva sobre la apetitiva, con respecto a la necesidad de responder de forma rápida, aunque poco precisa, dada la posibilidad de daño.

Los resultados de este estudio muestran que las imágenes del IAPS generan estados emocionales similares en los diferentes países (correlaciones positivas y significativas entre países en las tres dimensiones de la emoción) y que pueden ser utilizadas en población mexicana para el estudio de las emociones y la motivación.

Finalmente, algunas limitaciones deben ser tenidas en cuenta, pese a que este estudio se llevó a cabo en estudiantes universitarios, las investigaciones realizadas en diferentes países han utilizado poblaciones similares (Dufey et al., 2011; Gantiva et al., 2011; Lang et al., 1999; Lang et al., 2008; Moltó et al., 1999; Silva, 2011; Verschuere et al., 2001; Vila et al., 2001), y los resultados han demostrado ser confiables como punto de referencia para llevar a cabo investigaciones posteriores en poblaciones específicas, por ejemplo consumidores de sustancias psicoactivas (Gantiva, Rodriguez, Arias, \& Rubio, 2012; Muñoz et al., 2009), personas agresivas (Gantiva, Díaz, Ospina, Gutierrez, \& Romo-Gonzalez, 2014), con niveles significativos de ansiedad 
(Lang, Davis, \& Öhman, 2000), depresión (Taubitz, Robinson, \& Larson, 2013), entre otras.

\section{Conclusiones}

Los resultados de la presente investigación muestran que las imágenes de los conjuntos 13, 14, 19 y 20 pueden ser utilizadas en población mexicana para el estudio objetivo de las emociones, dado que estas imágenes generan respuestas emocionales con diferentes grados de valencia (apetitiva, neutral y aversiva), arousal (alto, medio y bajo) y dominancia (alta, media y baja).

\section{Referencias}

Bradley, M. M. (2009). Natural selective attention: Orienting and emotion. Psychophysiology, 46(1), 1-11.

Bradley, M. M., Codispoti, M., Cuthbert, B. N., \& Lang, P. J. (2001). Emotion and motivation I: Defensive and appetitive reactions in picture processing. Emotion, 1(3), 276-298.

Bradley, M. M., Codispoti, M., Sabatinelli, D., \& Lang, P. (2001). Emotion and motivation II: sex differences in picture processing. Emotion, 1(3), 300-319.

Bradley, M. M., \& Lang, P. J. (1994). Measuring emotion: The self-assessment manikin and the semantic differential. Journal of Behavior Therapy and Experimental Psychiatry, 25, 49-59.

Bradley, M. M., \& Lang, P. (2000). Affective reaction to acoustic stimuli. Psychophysiology, 37(2), 204-215.

Bradley, M. M., \& Lang, P. (2007). Emotion and motivation. En J. Cacioppo, L. Tassinary, \& G. Berntson (Eds.), The Handbook of Psychophysiology. Nueva York: Cambridge University Press.

Chayo-Dichy, R., Velez-Garcia, A. E., AriasGarcia, N., Castillo-Parra, G., \& OstroskySolis, F. (2003). Valencia, activación, dominancia y contenido moral, ante estímulos visuales con contenido emocional y moral: un estudio en población mexicana.
Revista Española de Neuropsicología, 5(3-4), 213-225.

Dufey, M., Fernández, A. M., \& Mayol, R. (2011). Adding support to cross-cultural emotional assessment: Validation of the International Affective Picture System in a Chilean sample. Universitas Psychologica, 10(2), 521-533.

Gantiva, C., Díaz, D., Ospina, I., Gutierrez, A., \& Romo-Gonzalez, T. (2014). Modulation of the startle response in verbal aggressors: differences among stimuli with distinct affective social content. Psychology $\mathbb{E}$ Neuroscience, 7(2), 221-225. https://doi.org $/ 10.3922 /$ j.psns.2014.016

Gantiva, C., Guerra, P., \& Vila, J. (2011). Validación colombiana del sistema internacional de imágenes afectivas: evidencias del origen transcultural de la emoción. Acta Colombiana de Psicología, 14(2), 103-111.

Gantiva, C., Rodriguez, M., Arias, M., \& Rubio, E. (2012). Dimensiones de la emocion durante el proceso de abandono del consumo de tabaco: un apoyo a la visión motivacional del cambio. Perspectiva Psicologica, 8(2), 309-317.

Lang, P. J. (1995). The emotion probe: Studies of motivation and attention. American Psychologist, 50(5), 372-385.

Lang, P. J. (2010). Emotion and motivation: Toward consensus definitions and a common research purpose. Emotion Review, 2(3), 229-233.

Lang, P. J., Bradley, M. M., \& Cuthbert, B. N. (1997). Motivated attention: Affect, activation and action. En P. J. Lang, R. F. Simons, \& M. F. Balaban (Eds.), Attention and Orienting: Sensory and Motivational Processes. (pp. 97-135). Hisllsdale: Lawrence Erlbaum Associates.

Lang, P. J., Bradley, M. M., \& Cuthbert, B. N. (1999). International affective picture system (IAPS): Affective ratings of pictures and instruction manual. Technical Report A-6. Gainesville: University of Florida.

Lang, P. J., Bradley, M. M., \& Cuthbert, B. N. (2008). International affective picture system 
(IAPS): Affective ratings of pictures and instruction manual. Technical Report A-8. Gainesville: University of Florida.

Lang, P. J., \& Davis, M. (2006). Emotion, motivation, and the brain: Reflex foundations in animal and human research. Progress in Brain Research, 156, 3-34.

Lang, P. J., Davis, M., \& Öhman, A. (2000). Fear and anxiety: animal models and human psychophysiology. Journal of Affective Disorders, 61 (3), 137-159.

LeDoux, J. (2000). Cognitive-emotional interaction: Listen to the brain. En R. D. Lane, \& L. Nadel (Eds.), Cognitive neuroscience of emotion (pp. 129-155). Nueva York: Oxford University Press.

Moltó, J., Montañes, S., Poy, R., Segarra, P., Pastor, M. C., Tormo, M. P., . . . \& Vila, J. (1999). Un nuevo método para el estudio experimental de las emociones. El International Affective Picture System (IAPS): Adaptación española. Revista de Psicología General y Aplicada, 52(1), 55-87.

Muñoz, M., Viedma-del-Jesús, M., FernándezSantaella, M., Peralta-Ramírez, M., Cepeda-Benito, A., \& Vila, J. (2009). Assessment of tobacco craving by means of the affective image visualization paradigm. Motivation and Emotion, 34(1), 93-103.

OCDE. (2014). Better Life Index. Recuperado de: http://stats.oecd.org/Index.aspx?DataS etCode $=$ BLI

Silva, J. (2011). International Affective Picture System (IAPS) in Chile: A cross-cultural adaptation and validation study. Terapia Psicológica, 29(2), 251-258.

Taubitz, L. E., Robinson, J. S., \& Larson, C. L. (2013). Modulation of the startle reflex across time by unpleasant pictures distinguishes dysphoric from nondysphoric women. International Journal of Psychophysiology, 87(2), 124-129. https://do i.org/10.1016/j.ijpsycho.2012.11.002.

Verschuere, B., Crombez, G., \& Koster, E. (2001). The internation affective picture system: a Flemish validation study. Psychologica Belgica, 41 (4), 2005-2217.
Vila, J., \& Fernández-Santaella, M. C. (2005). Tratamientos psicológicos: La perspectiva experimental. Madrid: Pirámide.

Vila, J., Sánchez, M., Ramírez, I., Fernández, M., Cobos, P., Rodríguez, S., . . .\& Moltó, J. (2001). El sistema internacional de imágenes afectivas (IAPS): Adaptación española. Segunda parte. Revista de Psicología General y Aplicada, 54(4), 635-657.

\section{Notas}

* Artículo de investigación. 